\title{
Comparison of four methods to evaluate sperm DNA integrity between mouse caput and cauda epididymidis
}

\begin{abstract}
Serafín Pérez-Cerezales, Alberto Miranda and Alfonso Gutiérrez-Adán
It is well known that transit through the epididymis involves an increase in the compaction of sperm chromatin, which acquires fully condensed status at the caput epididymidis. The purpose of this study was to compare the terminal deoxyribonucleotidyl transferase-mediated dUTP nick end-labelling (TUNEL) assay, the comet assay, the sperm chromatin structure assay (SCSA) and the sperm chromatin dispersion (SCD) test by analysing spermatozoa from the caput and cauda epididymidis in order to demonstrate the ability of each technique to discriminate between different degrees of sperm maturity related to chromatin compaction and DNA fragmentation. Our results suggest that some populations of DNA-fragmented spermatozoa associated with immature sperm can only be identified using the comet assay and the SCSA but not with the SCD test or the TUNEL assay.
\end{abstract}

Asian Journal of Andrology (2012) 14, 335-337; doi:10.1038/aja.2011.119; published online 17 October 2011

Keywords: DNA damage; mouse epididymis; sperm chromatin; sperm maturation; the comet assay; the sperm chromatin dispersion test; the sperm chromatin structure assay; TUNEL

\section{INTRODUCTION}

Sperm DNA fragmentation is gaining interest as a potential cause of infertility, and several techniques to quantify DNA damage are now used in the clinical setting. These assays include terminal deoxyribonucleotidyl transferase-mediated dUTP nick end-labelling (TUNEL) and single-cell gel electrophoresis (the comet assay) for the direct detection of DNA fragmentation. ${ }^{1,2}$ Additionally, the sperm chromatin dispersion (SCD) test and the sperm chromatin structure assay (SCSA) can analyse the susceptibility of chromatin to the denaturation ${ }^{2,3}$ allowing a complete view of the status of the nucleus in terms of DNA integrity and chromatin compaction. Nevertheless, there is ample literature on these techniques presenting contradictory results in correlative analyses and in their ability to reveal and discriminate DNA-chromatin status after exposure to different damaging agents, depending on the species and the disease

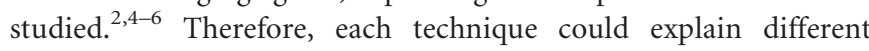
aspects of sperm chromatin status.

Spermatozoa acquire their final maturation status during their passage through the epididymis. In eutherians, during this maturational process, chromatin acquires its final highly compact status through the formation of disulfide bridges between the protamines. ${ }^{7}$ The objective of this work was to determine and characterize chromatin integrity and DNA compaction of mouse spermatozoa during their passage through the epididymis, to test the ability of the four techniques to evaluate chromatin compaction and integrity and to discriminate the different degrees of DNA fragmentation that could be related to the differential maturational status of the epididymal spermatozoa.

\section{MATERIALS AND METHODS}

Sperm from cauda and caput epididymidis was collected from ten 12week-old sexually mature CD1 male mice (Harlan, Oxon, UK) and prepared according to the requirements for each analytical technique. All animal experiments were performed in accordance with the Internal Institutional Animal Care and Use Committee of the National Institute for Agricultural and Food Research and Technology (Madrid, Spain). Sperm membrane integrity was evaluated by using the fluorescence 'live/dead kit' (Molecular Probes, Eugene, OR, USA) containing propidium iodide (PI) and SYBR-14.

For the SCD test, samples were processed by following the protocol included with the SPERM-HALOMAX-Mus-FF kit (ChomaCell SL, Madrid, Spain). Spermatozoa were classified as with or without a halo; the percentage of spermatozoa with a halo was recorded for each animal and epididymal section.

For the TUNEL assay, fragmented DNA was nick end-labelled with tetramethylrhodamine-conjugated dUTP by a terminal transferase (In Situ Cell Death Detection Kit; Roche Molecular Biochemicals, Sant Cugat del Valles, Spain) for $1 \mathrm{~h}$ at $37{ }^{\circ} \mathrm{C}$ in the dark after chromatin denaturation according to the manufacturer's instructions. ${ }^{8}$

The neutral comet assay was performed as described by Olive and Banath ${ }^{9}$ to determine the percentage of DNA fragmentation in each spermatozoa.

The SCSA was performed according to the inventor's instructions ${ }^{3}$ using flow cytometry (Beckman Coulter, Brea, CA, USA).

The samples processed for membrane integrity, the SCD test, and the TUNEL and comet assays were evaluated using a fluorescence

Department of Animal Reproduction, National Institute for Agricultural and Food Research and Technology, Madrid 28040, Spain Correspondence: Dr S Pérez-Cerezales (sperc@unileon.es)

Received: 29 March 2011; Revised: 12 May 2011; Accepted: 2 June 2011; Published online: 17 October 2011 
microscope equipped with appropriate filters (Optiphot-2; Nikon, Lijnden, The Netherlands).

Statistical analysis was performed with SPSS 14.0 software (IBM, New York, USA) for Windows. The results were expressed as means \pm s.e.m. and compared and analysed using one-way ANOVA followed by the Bonferroni post hoc test. Significance was set at $P<0.05$.

\section{RESULTS}

PI/SYBR-14 staining revealed a high permeability of the spermatozoa membrane in the caput epididymidis, allowing PI penetration into $50.4 \% \pm 3.2 \%$ of cells, whereas in the cauda epididymidis, the PI permeability was lower at $24.5 \% \pm 4.0 \%$ (Figure 1a).

Analyses of chromatin with the SCD test and the TUNEL assay did not reveal any differences between caput and cauda epididymal spermatozoa and indicated low percentages of spermatozoa with chromatin dispersion and DNA fragmentation (Figure 1b). However, the neutral comet assay showed a greater presence of double DNA strand breaks in the caput epididymal spermatozoa $(8.1 \% \pm 0.7 \%$ with DNA in the comet tail) than in the cauda $(5.5 \% \pm 0.3 \%)$ (Figure $1 \mathbf{b})$.

This trend was maintained in the results obtained with the SCSA. Flow cytometric analysis revealed a higher percentage of immature cells, detected as cells showing high DNA stainability (\% HDS) and a higher percentage of DNA-damaged cells, defined as DNA fragmentation index $(\% \mathrm{DFI})$ in the caput $(19.5 \% \pm 3.1 \%$ and $16.2 \% \pm 4.6 \%$, respectively) than in the cauda $(2.5 \% \pm 0.4 \%$ and $5.8 \% \pm 1.0 \%$, respectively) (Figure 2 ).

A significant positive correlation was found between the percentage of comet tail DNA determined with the neutral comet assay (percentage of fragmented DNA caused by double-strand breakage) and the percentage of immature cells (\% HDS) obtained with the SCSA (Figure 3a) (correlation coefficient of 0.64). Furthermore, both SCSA parameters (\% HDS and \% DFI) were directly correlated with each other (Figure 3b) (correlation coefficient of 0.51). Importantly, comet tail DNA, which is considered to be a direct measure of DNA fragmentation, was not correlated with \% DFI (correlation coefficient of 0.074 ), which is usually considered to be representative of a cell population with fragmented DNA.
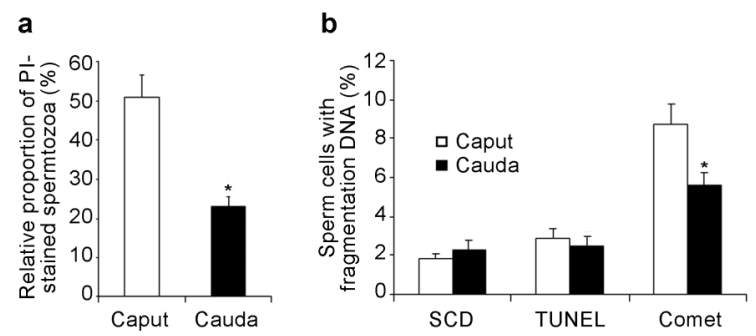

Figure 1 Assessment of plasma membrane and DNA integrity. (a) Evaluation of sperm membrane integrity. Histograms show the incorporation of propidium iodide (PI) into spermatozoa from the caput and cauda epididymidis. (b) Evaluation of sperm DNA fragmentation from the Halomax detection assay, based on the sperm chromatin dispersion (SCD) test and the terminal deoxyribonucleotidyl transferase-mediated dUTP nick end-labelling (TUNEL) and neutral comet assays (percentage of comet tail DNA). Values are expressed as mean \pm s.e.m. $* P<0.05$, compared with caput epididymidis.

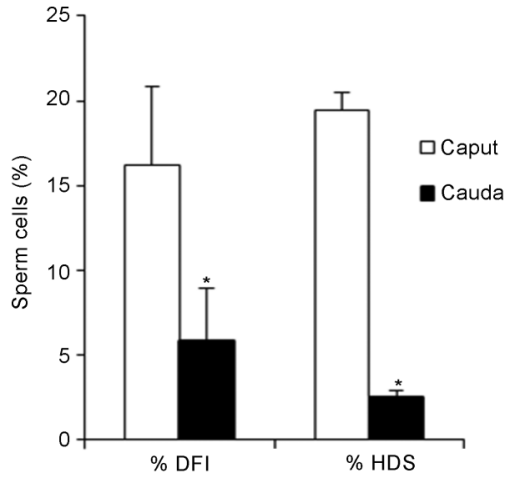

Figure $2 \%$ DNA fragmentation index (\% DFI) and cells with high DNA stainability (\% HDS), as obtained by the sperm chromatin structure assay (SCSA) for caput and cauda epididymal spermatozoa. Values are expressed as mean \pm s.e.m. ${ }^{*} P<0.05$, compared with caput epididymidis.

\section{DISCUSSION}

Several studies pointed out that the different techniques developed for the evaluation of sperm DNA fragmentation could explain different aspects of sperm chromatin status. ${ }^{5,6}$ In this study, the immaturity of the mouse spermatozoa in the caput epididymis was indicated based on the higher permeability of the membranes to PI, as reported by other authors. ${ }^{10}$ Sperm chromatin undergoes the formation of disulfide bridges between protamines during transit through the epididymis, increasing the compaction of the genetic material. ${ }^{11}$ Our results showed that neither the TUNEL assay nor the SCD test was able to detect differences between caput and cauda epididymal spermatozoa, which both showed low levels of DNA fragmentation and chromatin dispersion. These results were different from those obtained with the comet assay and the SCSA, which revealed different maturational stages of spermatozoa, with more DNA fragmentation and less chromatin compaction in the caput than in the cauda epididymal spermatozoa. Both the TUNEL assay and the SCD test probably require more marked DNA and chromatin damage before they can detect these changes, indicating that they have lower sensitivity than the SCSA and comet assay, or as has been suggested by other authors, these techniques could provide different information concerning chromatin status. ${ }^{4,6}$

The correlation between comet tail DNA HDS and DFI HDS shows the linkage between chromatin compaction and DNA
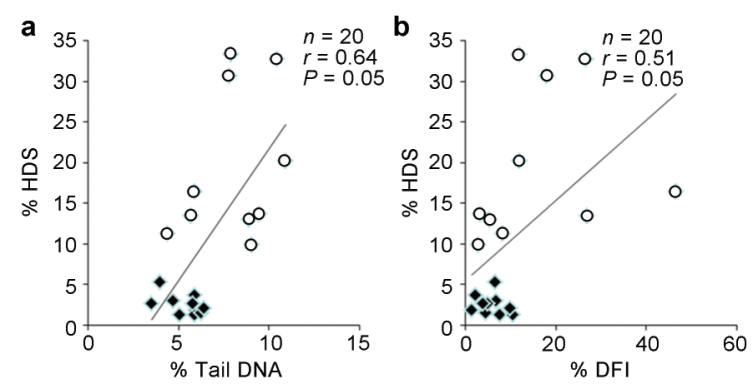

Figure 3 Correlation between Comet assay and SCSA parameters. (a) Correlation between the percentage of comet tail DNA and high DNA stainability (\% HDS) cells. (b) Correlation between the \% DNA fragmentation index (\% DFI) and (\% HDS) cells. Open circles represent cauda spermatozoa, and squares represent caput spermatozoa. 
fragmentation, defining the maturational status of the spermatozoa in the epididymis.

Interestingly, no correlation was found between comet tail DNA and DFI, parameters that theoretically indicate cells with fragmented DNA. This observation suggests that when young and healthy animals are analysed, DFI represents cells with a small amount of DNA fragmentation, reflecting an intermediate degree of chromatin compaction. We propose that this population could remain at an intermediate point during disulphide bridge formation, producing less green fluorescence than HDS (owing to the high degree of compaction that makes DNA staining difficult), but greater red fluorescence than the main population (owing to low compaction, which makes chromatin more susceptible to denaturation under acidic conditions).

\section{CONCLUSION}

Both SCSA and the neutral comet assay were able to distinguish the status of the sperm chromatin in cauda and caput epididymidis, whereas the SCD test and the TUNEL assay demonstrated insufficient resolving power to distinguish these differences.

\section{AUTHOR CONTRIBUTIONS}

SP-C and AG-A defined the research theme, designed the methods and experiments, and analysed the data. SP-C conducted the laboratory experiments. SP-C, AM and AG-A contributed to writing the paper and approving the manuscript

\section{COMPETING FINANCIAL INTERESTS}

The authors declare no competing financial interests.

\section{ACKNOWLEDGMENTS}

This work was funded by grant AGL2009-11358 from the Spanish Ministry of Science and Innovation.

1 Collins AR, Oscoz AA, Brunborg G, Gaivao I, Giovannelli L et al. The comet assay: topical issues. Mutagenesis 2008; 23: 143-51.

2 Henkel R, Hoogendijk CF, Bouic PJ, Kruger TF. TUNEL assay and SCSA determine different aspects of sperm DNA damage. Andrologia 2010; 42: 305-13.

3 Evenson DP, Melamed MR. Rapid analysis of normal and abnormal cell types in human semen and testis biopsies by flow cytometry. J Histochem Cytochem 1983; 31: 248-53.

4 Martinez-Pastor F, Fernandez-Santos MR, Dominguez-Rebolledo AE, Esteso MC, Garde JJ. DNA status on thawed semen from fighting bull: a comparison between the SCD and the SCSA tests. Reprod Domest Anim 2009; 44: 424-31.

5 Chohan KR, Griffin JT, Lafromboise M, de Jonge CJ, Carrell DT. Comparison of chromatin assays for DNA fragmentation evaluation in human sperm. $J$ Androl 2006; 27: 53-9.

6 Garcia-Macias V, de Paz P, Martinez-Pastor F, Alvarez M, Gomes-Alves S et al. DNA fragmentation assessment by flow cytometry and Sperm-Bos-Halomax (bright-field microscopy and fluorescence microscopy) in bull sperm. Int J Androl 2007; 30: 8898.

7 Haidl G, Badura B, Schill WB. Function of human epididymal spermatozoa. J Androl 1994; 15 Suppl: 23S-7S.

8 Perez-Crespo M, Pintado B, Gutierrez-Adan A. Scrotal heat stress effects on sperm viability, sperm DNA integrity, and the offspring sex ratio in mice. Mol Reprod Dev 2008; 75: 40-7.

9 Olive PL, Banath JP. The comet assay: a method to measure DNA damage in individual cells. Nat Protoc 2006; 1: 23-9.

10 Jones R. Plasma membrane structure and remodelling during sperm maturation in the epididymis. J Reprod Fertil Supp/ 1998; 53: 73-84

11 Golan R, Cooper TG, Oschry Y, Oberpenning F, Schulze H et al. Changes in chromatin condensation of human spermatozoa during epididymal transit as determined by flow cytometry. Hum Reprod 1996; 11: 1457-62. 